\title{
RELAÇÃO ENTRE DEPRESSÃO E POSTURA DE MULHERES MASTECTOMIZADAS
}

Edna Maria do Carmo ${ }^{1}$, Talita Ferreira Turatti ${ }^{1}$, Mário Hissamitsu Tarumoto ${ }^{2}$, Alessandra Madia Mantovani ${ }^{1}$, Fernanda Stellutti Magrini Pachioni ${ }^{1}$, Nathália Ulices Savian ${ }^{1}$, Célia Aparecida Stellutti Pachioni ${ }^{1}$, Dalva Minonroze Albuquerque Ferreira ${ }^{1}$, Cristina Elena Prado Teles Fregonesi ${ }^{1}$

Universidade Estadual Paulista, FCT, ${ }^{1}$ Departamento de Fisioterapia, ${ }^{2}$ Departamento de Matemática, Estatística e Computação, Presidente Prudente, SP. e-mail: edna@fct.unesp.br

\section{RESUMO}

A mastectomia é uma técnica cirúrgica necessária no tratamento do câncer de mama, porém pode causar sensações de estresse, gerando ansiedade e depressão. Esses fatores são responsáveis por desequilíbrios no organismo, como mialgias e alterações posturais. O objetivo foi verificar se há relação entre a depressão e a postura de mulheres mastectomizadas. Participaram deste estudo 40 mulheres, de 40 a 70 anos, subdivididas em grupo mastectomia - GM ( $n=20)$ composto por mastectomizadas e grupo controle - GC $(n=20)$ com mulheres sem a enfermidade. A coleta de dados baseou-se na tomada de fotografias, inserção destas imagens digitais no Software de Avaliação Postural (SAPO) e posterior análise postural. Para avaliação do estado depressivo foi utilizado o Inventário de Depressão de Beck. Constatou-se a existência de assimetrias corporais comuns no GM como elevação de ombro, anteriorização de cabeça, inclinação posterior de tronco e retroversão pélvica. Esse estudo conclui que a postura corporal e a depressão não estão associadas à presença da mastectomia.

Palavras-chave: postura, neoplasias da mama, depressão, mastectomia, fisioterapia.

\section{RELATIONSHIP BETWEEN DEPRESSION AND POSTURE OF MASTECTOMIZED WOMEN}

\begin{abstract}
The study objective was to determine whether there is a relationship between depression and the posture of women who underwent mastectomy. The study included 40 women, 40 to 70 years, divided into an experimental group - GE $(n=20)$ comprised of mastectomy and control group - CG $(n=20)$ with women without the disease. Data collection was based on taking photographs, digital images of these insertions in Software Postural Assessment (SAPO) and posterior postural analysis. To assess depressive state was used Beck Depression Inventory and the comparison between these women's groups were significant. We found the existence of common bodily asymmetries in GM as shoulder elevation, anterior head, posterior inclination of trunk and pelvic tilt. This study concludes that the body posture and depression are not associated with the presence of mastectomy.
\end{abstract}

Keywords: posture, breast neoplasms, depression, mastectomy, physiotherapy. 


\section{INTRODUÇÃO}

As cirurgias de mastectomias podem trazer diversas alterações funcionais, sequelas e complicações para as pacientes submetidas a essa conduta terapêutica, tais como: má cicatrização, fibrose tecidual, síndrome da mama fantasma, alterações respiratórias, diminuição da amplitude de movimento, dores e edema do membro superior, além de retrações cicatriciais e quelóides, o que limita a amplitude de movimento do membro superior homolateral à intervenção cirúrgica e pode ocorrer grandes mudanças na postura corporal de mulheres mastectomizadas em comparação com mulheres saudáveis na mesma faixa etária ${ }^{1,2,3}$.

A avaliação postural do indivíduo é um exame indispensável na rotina fisioterapêutica, pois tem o propósito de analisar e quantificar os desvios e, a partir dessas informações, proporcionar a melhor conduta a ser desenvolvida, elegendo os métodos terapêuticos mais indicados para cada alteração específica ${ }^{4}$. Para uma avaliação postural fidedigna e confiável, além da análise da simetria postural, se pode dispor de recursos informatizados, como é o caso da fotogrametria computadorizada, que é um método eficaz e seguro na avaliação, análise e quantificação das alterações posturais $^{5,6}$. Porém, estudos de avaliação postural com mulheres mastectomizadas por meio de fotogrametria computadorizada ainda são escassos na literatura ${ }^{7,8}$.

Além disso, as mulheres mastectomizadas ainda podem apresentar sintomas afetivos devido a experiência emocional dolorosa gerando a chamada "postura depressiva" 9

Diante da diversidade e excassez de resultados de avaliação postural em mulheres mastectomizadas e a associação com o estado emocional das mesmas. O objetivo foi verificar se há relação entre a depressão e a postura de mulheres mastectomizadas.

\section{MÉTODOS}

Trata-se de um estudo do tipo transversal com a composição da amostra por conveniência, no qual foram avaliadas 40 mulheres, faixa etária de 40 a 70 anos, divididas em dois grupos: Grupo Mastectomia (GM) - composto por 20 mulheres submetidas a tratamento cirúrgico de câncer de mama, atendidas periodicamente num Centro de Atendimento em Fisioterapia e Reabilitação em Presidente Prudente; e Grupo Controle (GC) - composto por 20 mulheres sem história prévia de câncer de mama.

Foram definidos como critérios de inclusão mulheres sem restrição de idade, submetidas ao procedimento cirúrgico pela técnica de mastectomia unilateral para o 
grupo GM e mulheres sem histórico de câncer para o GC. Como critérios de exclusão, mulheres com diagnóstico prévio de alguma alteração postural comprovada clinicamente por médico especialista, e aquelas incapazes de permanecer em posição ortostática.

Todas foram devidamente esclarecidas sobre os objetivos e procedimentos do estudo e assinaram o "Termo de Consentimento Livre e Esclarecido". O estudo foi aprovado pelo Comitê de Ética em Pesquisa da FCT/UNESP, protocolo $n^{\circ} 45 / 2009$, cujos procedimentos obedecem aos Critérios da Ética em Pesquisa com Seres Humanos conforme Resolução no. 196/96 do Conselho Nacional de Saúde.

Para a realização da avaliação postural os sujeitos foram fotografados com trajes de banho, com base de sustentação livre. Foi utilizada uma câmera fotográfica digital, apoiada sobre um tripé, distante 1,5 metro do sujeito e com altura correspondente a metade da estatura do mesmo. Em um aro retilíneo, perpendicular ao chão, foi colocado um fio de prumo demarcado com duas bolas grandes de isopor, distantes $70 \mathrm{~cm}$ uma da outra, para possibilitar a calibração do Software para Avaliação Postural (SAPO) ${ }^{9}$. Para garantir a mesma base de sustentação, foi realizada uma marcação podal em uma cartolina preta (40 x $40 \mathrm{~cm}$ ), para cada indivíduo, que era girada em $90^{\circ}$, na sequência da realização das fotografias, em vista anterior, lateral direita, posterior e lateral esquerda.

A escolha dos pontos anatômicos ocorreu em função de critérios do tutorial do próprio software o qual disponibiliza um protocolo modelo para possibilitar análises clínicas relevantes. Tais pontos foram marcados com bolas pequenas de isopor, fixadas com fita dupla face.

À medida que os dados de cada voluntária eram analisados no SAPO houve a necessidade de classificar cada variável de acordo com as alterações posturais correspondentes. Estas variáveis, por sua vez, foram abreviadas em siglas que fazem referência à vista e aos pontos anatômicos correspondentes (Tabela 1). 
Tabela 1. Alterações posturais com interpretação de cada variável, siglas e alterações posturais correspondentes.

\begin{tabular}{|c|c|c|c|c|c|}
\hline \multirow[t]{2}{*}{ Vistas } & Variáveis & $\begin{array}{l}\text { Pontos } \\
\text { Utilizados }\end{array}$ & Siglas & $\begin{array}{l}\text { Ângulos Positivos } \\
\text { (>1) }\end{array}$ & $\begin{array}{l}\text { Ângulos Negativos } \\
(<-1)\end{array}$ \\
\hline & $\begin{array}{l}\text { Alinhamento } \\
\text { Horizontal de } \\
\text { Cabeça }\end{array}$ & $\begin{array}{l}\text { Glabela/ Mento/ } \\
\text { Horizontal }\end{array}$ & VAAHC & Inclinação Direita & Inclinação Esquerda \\
\hline \multirow[t]{3}{*}{ Anterior } & $\begin{array}{l}\text { Alinhamento } \\
\text { Horizontal de } \\
\text { Acrômios }\end{array}$ & $\begin{array}{l}\text { Acrômio } \\
\text { direito/esquerdo/ } \\
\text { horizontal }\end{array}$ & VAAHA & Inclinação Direita & Inclinação Esquerda \\
\hline & $\begin{array}{l}\text { Alinhamento } \\
\text { Horizontal de } \\
\text { EIAS. }\end{array}$ & $\begin{array}{l}\text { Espinhas llíacas Antero- } \\
\text { Superiores/ Horizontal }\end{array}$ & VAAHES & Inclinação Direita & Inclinação Esquerda \\
\hline & $\begin{array}{l}\text { Alinhamento } \\
\text { Horizontal de } \\
\text { Acrômios e } \\
\text { EIAS. }\end{array}$ & $\begin{array}{l}\text { Acrômios/Espinhas } \\
\text { Ilíacas Antero Superiores }\end{array}$ & VAAHAES & Inclinação Direita & Inclinação Esquerda \\
\hline \multirow[t]{2}{*}{ Posterior } & $\begin{array}{l}\text { Alinhamento } \\
\text { Horizontal de } \\
\text { Escápulas em } \\
\text { relação a T3 }\end{array}$ & $\begin{array}{l}\text { Apófise Espinhosa T3/ } \\
\text { ângulos inferiores das } \\
\text { Escápulas/Horizontal }\end{array}$ & VPAHET3 & $\begin{array}{l}\text { Escápulas } \\
\text { Assimétricas }\end{array}$ & $\begin{array}{l}\text { Escápulas } \\
\text { Assimétricas }\end{array}$ \\
\hline & $\begin{array}{l}\text { Alinhamento } \\
\text { Horizontal de } \\
\text { Cabeça }\end{array}$ & $\begin{array}{l}\text { C7/Lóbulo da } \\
\text { orelha/Horizontal }\end{array}$ & VLDAHC & Flexão & Extensão \\
\hline \multirow[t]{4}{*}{$\begin{array}{l}\text { Lateral } \\
\text { Direita }\end{array}$} & $\begin{array}{l}\text { Alinhamento } \\
\text { Vertical de } \\
\text { Cabeça }\end{array}$ & $\begin{array}{l}\text { Acrômios/ Lóbulo da } \\
\text { orelha/Vertical }\end{array}$ & VLDAVC & Anteriorização & Posteriorização \\
\hline & $\begin{array}{l}\text { Alinhamento } \\
\text { Vertical de } \\
\text { Tronco }\end{array}$ & $\begin{array}{l}\text { Acrômio/ Trocânter } \\
\text { maior do fêmur/ Vertical }\end{array}$ & VLDAVT & $\begin{array}{l}\text { Inclinação } \\
\text { Anterior }\end{array}$ & Inclinação Posterior \\
\hline & $\begin{array}{l}\text { Alinhamento } \\
\text { Horizontal de } \\
\text { Pelve. }\end{array}$ & $\begin{array}{l}\text { Espinha ilíaca ântero- } \\
\text { superior/ Espinha ilíaca } \\
\text { pôstero-superior/ } \\
\text { Horizontal }\end{array}$ & VLDAHP & Anteversão & Retroversão \\
\hline & $\begin{array}{l}\text { Alinhamento } \\
\text { Horizontal de } \\
\text { Cabeça }\end{array}$ & $\begin{array}{l}\text { C7/Lóbulo da } \\
\text { orelha/Horizontal }\end{array}$ & VLEAHC & Extensão & Flexão \\
\hline \multirow[t]{3}{*}{$\begin{array}{l}\text { Lateral } \\
\text { Esquerda }\end{array}$} & $\begin{array}{l}\text { Alinhamento } \\
\text { Vertical de } \\
\text { Cabeça }\end{array}$ & $\begin{array}{l}\text { Acrômios/ Lóbulo da } \\
\text { orelha/ Vertical }\end{array}$ & VLEAVC & Posteriorização & Anteriorização \\
\hline & $\begin{array}{l}\text { Alinhamento } \\
\text { Vertical de } \\
\text { Tronco }\end{array}$ & $\begin{array}{l}\text { Acrômio/ Trocânter } \\
\text { maior do fêmur/ Vertical }\end{array}$ & VLEAVT & $\begin{array}{l}\text { Inclinação } \\
\text { Posterior }\end{array}$ & Inclinação Anterior \\
\hline & $\begin{array}{l}\text { Alinhamento } \\
\text { Horizontal de } \\
\text { Pelve. }\end{array}$ & $\begin{array}{l}\text { Espinha ilíaca ântero- } \\
\text { superior/ Espinha ilíaca } \\
\text { pôstero-superior/ } \\
\text { Horizontal }\end{array}$ & VLEAHP & Retroversão & Anteversão \\
\hline
\end{tabular}

Nota: Vista Anterior Alinhamento Horizontal de Cabeça (VAAHC); Vista Anterior Alinhamento Horizontal de Acrômios (VAAHA); Vista Anterior Alinhamento Horizontal de Espinha llíaca Antero-Superior (VAAHES); Vista Anterior Alinhamento Horizontal de Acrômios e Espinha llíaca Antero-Superior (VAAHAES); Vista Posterior Alinhamento Horizontal de Escápulas em relação a T3 (VPAHET3); Vista Lateral Direita Alinhamento Horizontal de Cabeça (VLDAHC); 
Vista Lateral Direita Alinhamento Vertical de Cabeça (VLDAVC); Vista Lateral Direita Alinhamento Vertical de Tronco (VLDAVT); Vista Lateral Direita Alinhamento Horizontal de Pelve (VLDHP); Vista Lateral Esquerda Alinhamento Horizontal de Cabeça (VLEAHC); Vista Lateral Esquerda Alinhamento Vertical de Cabeça (VLEAVC); Vista Lateral Esquerda Alinhamento Vertical de Tronco (VLEAVT); Vista Lateral Esquerda Alinhamento Horizontal de Pelve (VLEAHP).

Foram estipulados como valores de normalidade postural todo ângulo encontrado entre os valores de $-1^{\circ}$ e $1^{\circ}$, já que a possibilidade de encontrar apenas valores de normalidade com ângulos de $0^{\circ}$ seria muito remota.

Para a avaliação do estado emocional foi utilizado o Inventário de Depressão de Beck (IDB) ${ }^{10}$ composto por 21 itens, incluindo sintomas e atitudes, cuja intensidade varia de 0 a 3, com escore máximo de 63 . Os itens referem-se à tristeza, pessimismo, sensação de fracasso, irritabilidade, retração social, entre outros. Foi analisado como o sujeito está se sentindo na última semana. O "Centro de Terapia Cognitiva de Beck" recomenda a seguinte pontuação: menor que 10: sem depressão ou depressão mínima; de 10 a 18: depressão de leve a moderada; de 19 a 29: depressão de moderada a grave e de 30 a 63: depressão grave.

Foi realizada uma análise descritiva para expor os resultados obtidos. A apresentação das variáveis mensuradas foi feita através de tabelas e figuras (média \pm desvio padrão).

Para a análise estatística, primeiramente, foi verificado se as variáveis deste estudo seguiam uma Distribuição
Normal, com o Teste de Shapiro - Wilks, ao nível de significância de 5\%, em ambos os grupos. Os dados apresentaram distribuição normal para a maioria das variáveis, tanto do $\mathrm{GM}$, quanto do $\mathrm{GC}$, exceto as variáveis VLDAHC e VLDDAVT do GM e a variável VLEAHP do GC. Esse fato não afeta os resultados das análises posteriores, pois a técnica de análise a ser utilizada (ANOVA) é robusta, podendo ser utilizada mesmo nesta situação ${ }^{11}$.

Foi realizada comparação entre os grupos (GM e GC) para as variáveis posturais e para Inventário de Depressão de Beck por meio do teste $t$ para dados independentes. Os valores percentuais das frequências de alterações posturais encontradas em cada um dos grupos, foram analisados por meio do Teste Exato de Fisher. Foi utilizado o nível de significância de $5 \%$.

\section{RESULTADOS}

A amostra de mulheres do grupo mastectomizadas $(n=20)$ apresentou idade média de 58,1 $\pm 10,2$ anos, peso corporal médio de $74,5 \pm 15,0 \mathrm{Kg}$. No grupo controle $(n=20)$ a idade média foi $62,35 \pm 8,02$ anos e o peso corporal médio $71,45 \pm 17,05 \mathrm{Kg}$. 
A aplicação do IDB proporcionou pontuações decorrentes da soma das alternativas escolhidas pelas voluntárias e estas pontuações, por sua vez, geraram classificações crescentes do estado depressivo. De forma isolada, os dados obtidos com a aplicação deste questionário, não evidenciou diferença significante entre os grupos. Na Figura 1 é apresentado o percentual observado de pacientes nas diferentes classificações de graus de depressão em cada grupo.

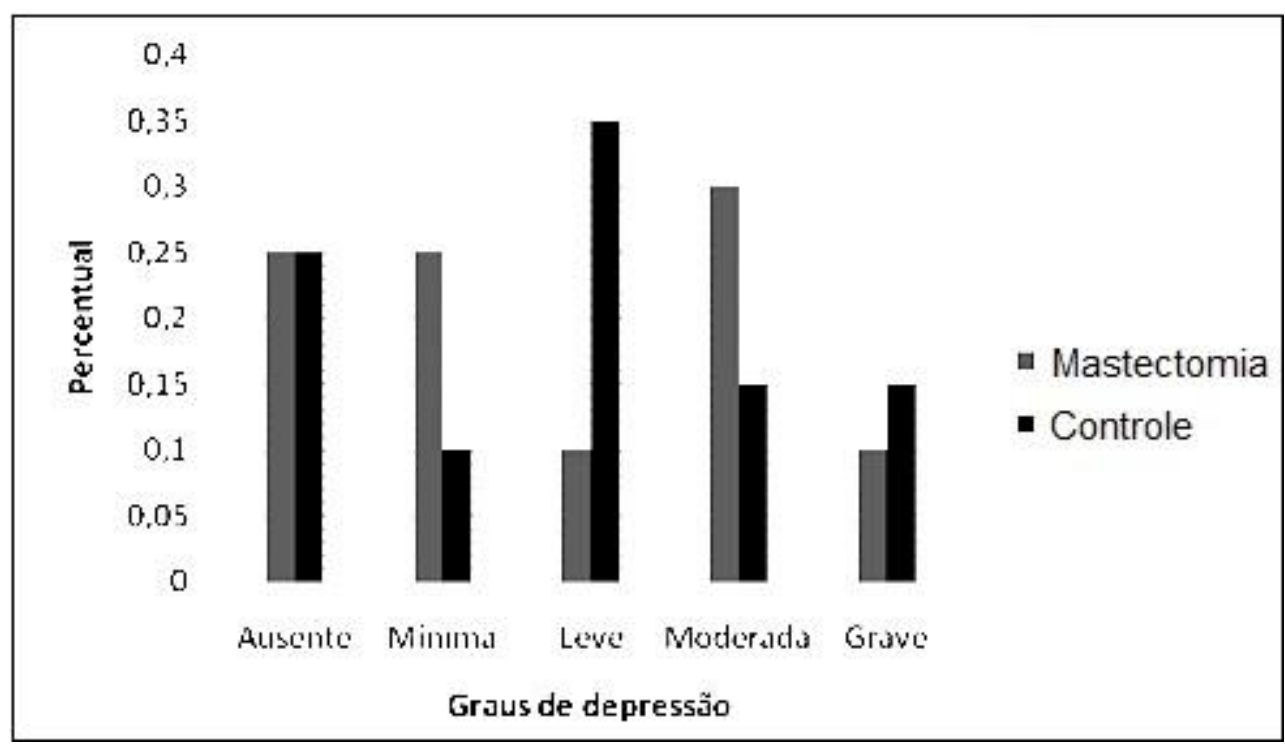

Figura 1. Graus de depressão. Valores em percentual das classificações de graus de depressão predominantes em cada grupo (Mastectomizadas e Controle).

Em relação às alterações posturais não foi detectada diferença significante na comparação entre os grupos, para nenhuma das variáveis estudadas.
A Tabela 2 descreve, em valores percentuais, as frequências de alterações posturais encontradas em cada um dos grupos. 
Tabela 2. Variáveis posturais. Distribuição de frequências (percentual) de sujeitos por grupo para cada variável observada e tipo de desvio encontrado.

\begin{tabular}{|c|c|c|c|c|}
\hline \multirow[b]{2}{*}{ VARIÁVEIS } & \multirow[b]{2}{*}{ GRUPO } & \multicolumn{3}{|c|}{ TIPO DE DESVIO } \\
\hline & & $\begin{array}{c}\text { Ângulos Negativos } \\
(<-1)\end{array}$ & Normalidade $\left(-1^{\circ}<\mathrm{x}<1^{\circ}\right)$ & $\begin{array}{c}\text { Ângulos Positivos } \\
\left(>1^{\circ}\right)\end{array}$ \\
\hline \multirow[t]{3}{*}{ VAAHC } & Controle & $10(50,00)$ & $3(15,00)$ & $7(35,00)$ \\
\hline & Mastectomia & $5(25,00)$ & $8(40,00)$ & $7(35,00)$ \\
\hline & Total & $15(37,50)$ & $11(27,50)$ & $14(35,00)$ \\
\hline \multirow[t]{3}{*}{ VAAHA } & Controle & $4(20,00)$ & $5(25,00)$ & $11(55,00)$ \\
\hline & Mastectomia & $9(45,00)$ & $1(5,00)$ & $10(50,00)$ \\
\hline & Total & $13(32,50)$ & $6(15,00)$ & $21(52,50)$ \\
\hline \multirow[t]{3}{*}{ VAAHES } & Controle & $4(20,00)$ & $7(35,00)$ & $9(45,00)$ \\
\hline & Mastectomia & $4(20,00)$ & $10(50,00)$ & $6(30,00)$ \\
\hline & Total & $8(20,00)$ & $17(42,50)$ & $15(17,50)$ \\
\hline \multirow[t]{3}{*}{ VAAHAES } & Controle & $8(40,00)$ & $6(30,00)$ & $6(30,00)$ \\
\hline & Mastectomia & $7(35,00)$ & $5(25,00)$ & $8(40,00)$ \\
\hline & Total & $15(37,50)$ & $11(27,50)$ & $14(35,00)$ \\
\hline \multirow[t]{3}{*}{ VPAHET3 } & Controle & $8(40,00)$ & $0(0,00)$ & $12(60,00)$ \\
\hline & Mastectomia & $13(65,00)$ & $1(5,00)$ & $6(30,00)$ \\
\hline & Total & $21(52,50)$ & $1(2,50)$ & $18(45,00)$ \\
\hline \multirow[t]{3}{*}{ VLDAHC } & Controle & $0(0,00)$ & $0(0,00)$ & $20(100,00)$ \\
\hline & Mastectomia & $0(0,00)$ & $0(0,00)$ & $20(100,00)$ \\
\hline & Total & $0(0,00)$ & $0(0,00)$ & $40(100,00)$ \\
\hline \multirow[t]{3}{*}{ VLDAVC } & Controle & $1(5,00)$ & $1(5,00)$ & $18(90,00)$ \\
\hline & Mastectomia & $4(20,00)$ & $1(5,00)$ & $15(75,00)$ \\
\hline & Total & $5(12,50)$ & $2(5,00)$ & $33(82,50)$ \\
\hline \multirow[t]{3}{*}{ VLDAVT } & Controle & $16(80,00)$ & $2(10,00)$ & $2(10,00)$ \\
\hline & Mastectomia & $16(80,00)$ & $2(10,00)$ & $2(10,00)$ \\
\hline & Total & $32(80,00)$ & $4(10,00)$ & $4(10,00)$ \\
\hline \multirow[t]{3}{*}{ VLDAHP } & Controle & $19(95,00)$ & $1(5,00)$ & $0(0,00)$ \\
\hline & Mastectomia & $20(100,00)$ & $0(0,00)$ & $0(0,00)$ \\
\hline & Total & $20(50,00)$ & $20(50,00)$ & $0(0,00)$ \\
\hline \multirow[t]{3}{*}{ VLEAHC } & Controle & $0(0,00)$ & $0(0,00)$ & $20(100,00)$ \\
\hline & Mastectomia & $0(0,00)$ & $0(0,00)$ & $20(100,00)$ \\
\hline & Total & $0(0,00)$ & $0(0,00)$ & $40(100,00)$ \\
\hline \multirow[t]{3}{*}{ VLEAVC } & Controle & $2(10,00)$ & $0(0,00)$ & $18(90,00)$ \\
\hline & Mastectomia & $3(15,00)$ & $0(0,00)$ & $17(85,00)$ \\
\hline & Total & $5(12,50)$ & $0(0,00)$ & $35(87,50)$ \\
\hline \multirow[t]{3}{*}{ VLEAVT } & Controle & $16(80,00)$ & $1(5,00)$ & $3(15,00)$ \\
\hline & Mastectomia & $18(90,00)$ & $2(10,00)$ & $0(0,00)$ \\
\hline & Total & $3(85,00)$ & $3(7,50)$ & $3(7,50)$ \\
\hline \multirow[t]{3}{*}{ VLEAHP } & Controle & $20(100,00)$ & $0(0,00)$ & $0(0,00)$ \\
\hline & Mastectomia & $20(100,00)$ & $0(0,00)$ & $0(0,00)$ \\
\hline & Total & $40(100,00)$ & $0(0,00)$ & $0(0,00)$ \\
\hline
\end{tabular}


Nota: Vista Anterior Alinhamento Horizontal de Cabeça (VAAHC); Vista Anterior Alinhamento Horizontal de Acrômios (VAAHA); Vista Anterior Alinhamento Horizontal de Espinha Ilíaca Antero-Superior (VAAHES); Vista Anterior Alinhamento Horizontal de Acrômios e Espinha llíaca Antero-Superior (VAAHAES); Vista Posterior Alinhamento Horizontal de Escápulas em relação a T3 (VPAHET3); Vista Lateral Direita Alinhamento Horizontal de Cabeça (VLDAHC); Vista Lateral Direita Alinhamento Vertical de Cabeça (VLDAVC); Vista Lateral Direita Alinhamento Vertical de Tronco (VLDAVT); Vista Lateral Direita Alinhamento Horizontal de Pelve (VLDHP); Vista Lateral Esquerda Alinhamento Horizontal de Cabeça (VLEAHC); Vista Lateral Esquerda Alinhamento Vertical de Cabeça (VLEAVC); Vista Lateral Esquerda Alinhamento Vertical de Tronco (VLEAVT); Vista Lateral Esquerda Alinhamento Horizontal de Pelve (VLEAHP).

\section{Associação das alterações posturais com o estado emocional}

Todas as voluntárias apresentaram alterações posturais em algum dos aspectos analisados; por isso, foi possível traçar perfis que associassem às alterações posturais apresentadas, com o estado depressivo em que as mesmas foram classificadas. $\mathrm{Na}$ Tabela 3 é apresentada a distribuição de frequências relativas entre 0 tipo de incidência de depressão e a alteração postural, no entanto, pelo Teste Exato de Fisher as associações não foram significativas.

\section{DISCUSSÃO}

Os resultados deste estudo apontam para a diversidade de posicionamentos dos segmentos corporais e uma tendência de assimetria dos segmentos bilaterais, possibilitando afirmar que mulheres submetidas ao processo cirúrgico de mastectomia ou não, apresentam alterações posturais em diferentes escalas.

Analisando os dados podemos destacar algumas tendências posturais predominantes em pessoas depressivas de ambos os grupos. O posicionamento da cabeça em flexão e em anteriorização, o tronco em inclinação posterior e pelve em retroversão foram às alterações mais predominantes nos indivíduos com depressão de moderada a grave, sendo que, pessoas classificadas com depressão mínima ou leve também compartilharam destes mesmos aspectos alterados, em menor proporção.

O grupo de voluntárias mastectomizadas apresentou predominância de alterações posturais como, por exemplo, a variável VAAHA na qual $95 \%$ do GM apresentaram inclinação de ombro para um dos lados, sendo que, 50\% destas tinham seus ombros esquerdos mais elevados, enquanto que $45 \%$ apresentaram elevação do ombro direito. Estes dados convergem com os achados de Cardoso e Oliveira ${ }^{12}$ que, objetivando avaliar a postura corporal de cinco mulheres submetidas à mastectomia, por meio de imagens digitais e goniometria, observaram, dentre outras alterações posturais, a elevação de ombro nas cinco 
voluntárias avaliadas, sendo que três destas

apresentaram elevação do ombro

contralateral e duas apresentaram elevação

do ombro homolateral à cirurgia.

Tabela 3. Associação alteração postural e intensidade da depressão. Distribuição de frequências (percentual) de pacientes para cada tipo de depressão e as alterações posturais encontradas.

\begin{tabular}{|c|c|c|c|c|c|c|}
\hline \multirow[t]{2}{*}{ ALTERAÇÃO POSTURAL } & \multicolumn{5}{|c|}{ INCIDÊNCIA DE DEPRESSÃO (\%) } & \multirow[t]{2}{*}{ TOTAL } \\
\hline & Ausente & Mínima & Leve & Moderada & Grave & \\
\hline $\begin{array}{l}\text { Inclinação de cabeça para } \\
\text { esquerda }\end{array}$ & 7,5 & 7,5 & 7,5 & 10,0 & 5,0 & 37,5 \\
\hline Normal & 5,0 & 5,0 & 5,0 & 10,0 & 2,5 & 27,5 \\
\hline $\begin{array}{l}\text { Inclinação de cabeça para } \\
\text { direita }\end{array}$ & 12,5 & 5,0 & 10,0 & 2,5 & 5,0 & 35,0 \\
\hline Elevação de ombro esquerdo & 7,5 & 7,5 & 5,0 & 10,0 & 2,5 & 32,5 \\
\hline Normal & 5,0 & 0 & 5,0 & 2,5 & 2,5 & 15,0 \\
\hline Elevação de ombro direito & 12,5 & 10,0 & 12,5 & 10,0 & 7,5 & 52,5 \\
\hline $\begin{array}{l}\text { Inclinação de } \\
\text { esquerda }\end{array}$ & 7,5 & 2,5 & 5,0 & 5,0 & 0 & 20,0 \\
\hline Normal & 12,5 & 7,5 & 10,0 & 10,0 & 2,5 & 42,5 \\
\hline Inclinação de EIAS para direita & 5,0 & 7,5 & 7,5 & 7,5 & 10,0 & 37,5 \\
\hline $\begin{array}{l}\text { Incl. Acrômios e EIAS para } \\
\text { esquerda }\end{array}$ & 15,0 & 7,5 & 7,5 & 7,5 & 0 & 37,5 \\
\hline Normal & 2,5 & 0 & 12,5 & 5,0 & 7,5 & 27,5 \\
\hline $\begin{array}{l}\text { Incl. Acrômios e EIAS para } \\
\text { direita }\end{array}$ & 7,5 & 10,0 & 2,5 & 10,0 & 5,0 & 35,0 \\
\hline $\begin{array}{l}\text { Assimetria de } \quad \text { Escápula } \\
\text { Esquerda }\end{array}$ & 17,5 & 5,0 & 12,5 & 12,5 & 5,0 & 52,5 \\
\hline Normal & 0 & 2,5 & 0 & 0 & 0 & 2,5 \\
\hline Assimetria de Escápula Direita & 7,5 & 10,0 & 10,0 & 10,0 & 7,5 & 45,0 \\
\hline Flexão de Cabeça & 25,0 & 17,5 & 22,5 & 22,5 & 12,5 & 100,0 \\
\hline Normal & 0 & 0 & 0 & 0 & 0 & 0 \\
\hline Extensão de Cabeça & 0 & 0 & 0 & 0 & 0 & 0 \\
\hline Inclinação Posterior de Tronco & 17,5 & 12,5 & 20,0 & 17,5 & 12,5 & 80,0 \\
\hline Normal & 5,0 & 2,5 & 0 & 2,5 & 0 & 10,0 \\
\hline Inclinação Anterior de Tronco & 2,5 & 2,5 & 2,5 & 2,5 & 0 & 10,0 \\
\hline Posteriorização & 2,5 & 2,5 & 2,5 & 5,0 & 0 & 12,5 \\
\hline Normal & 2,5 & 0 & 0 & 2,5 & 5,0 & 5,0 \\
\hline Anteriorização & 20,0 & 15,0 & 20,0 & 15,0 & 12,5 & 82,5 \\
\hline Retroversão Pélvica & 22,5 & 17,5 & 22,5 & 22,5 & 12,5 & 97,5 \\
\hline Normal & 2,5 & 0 & 0 & 0 & 0 & 2,5 \\
\hline Anteversão Pélvica & 0 & 0 & 0 & 0 & 0 & 0 \\
\hline
\end{tabular}

Com relação a essa variável, buscouse compreender que caso a elevação de ombro ocorra no lado homolateral à cirurgia, este tipo de alteração pode estar relacionada com a falta de peso da mama; e por outro lado, quando a elevação de ombro ocorre no lado contralateral à cirurgia, esta alteração pode estar relacionada com complicações 
pós-cirúrgicas, como a aderência cicatricial do local ou lesões músculoesqueléticas. Silva e Rodrigues $^{13}$ afirmam que o ombro homolateral à cirurgia pode se elevar e girar internamente, abduzindo a escápula e provocando contraturas musculares em mulheres submetidas à cirurgia de mastectomia.

Por meio das variáveis VLDAHP e VLEAHP, as quais podem indicar anteversão, retroversão ou normalidade pélvica, observou-se que todas as voluntárias do GM apresentaram alteração postural em retroversão pélvica. No estudo de Cardoso e Oliveira $^{12}$, esta variável também foi descrita, mas constatou-se que apenas uma paciente, dentre cinco voluntárias avaliadas, apresentou retroversão pélvica e, provavelmente devido ao pequeno número de indivíduos analisados, esta variável não foi discutida quanto à sua origem. Porém, Canales $^{5}$ apresentou resultados que demonstraram que indivíduos depressivos em maior grau podem adquirir uma postura corporal característica em retroversão pélvica e anteriorização de cabeça. Com isso, esta alteração postural, no presente estudo, pode estar associada às sequelas físicas póscirúrgicas e/ou às sequelas emocionais decorrentes desta vivência.

A variável VPAHET3 esteve alterada em 95\% das voluntárias do GM. Este tipo de alteração tem uma relação direta com o posicionamento dos ombros, visto que, estas estruturas e seus componentes ósseos são acoplados de maneira que seus movimentos e posicionamentos ocorram em conjunto.

A observação empírica e a grande ocorrência de alterações posturais, tanto no GM quanto no $\mathrm{GC}$, denunciam que o padrão de referência postural simétrico não ocorre na população, confirmando que mesmo pessoas que não referem nenhuma dor ou alteração no sistema músculo-esquelético, podem apresentar alterações na postura. Ferreira ${ }^{9}$ discute que a falta de um padrão que se aproxime da realidade em termos de alinhamento postural gera problemas, como por exemplo, a dificuldade de poder comparar os dados advindos de uma avaliação precisa da postura do paciente e da evolução do tratamento fisioterapêutico, com um padrão de referência plausível.

Ainda assim, uma observação detalhada da postura corporal de um grupo de pessoas se faz importante quando se pretende caracterizar e compreender a existência de alterações quanto a sua origem e complexidade. Sendo assim, Ferreira e Kappaun $^{14}$ realizaram um estudo com o objetivo de conhecer a imagem corporal de mulheres que realizaram a cirurgia de retirada da mama, através de sua história de vida. Uma vez que o câncer de mama é entendido como agente estressor e a mastectomia como uma mutilação, buscou-se 
perceber como essas mulheres se representam e se relacionam consigo mesmas e com os outros. Os autores entrevistaram três mulheres e nos temas referidos durante as entrevistas, essas mulheres relataram o medo da morte, sensação de mutilação, receio e algumas dificuldades com situações que envolvessem a exposição do corpo, mas também o alívio pela compreensão de que a doença foi extirpada. A escala de satisfação corporal quantificou o nível de insatisfação corporal apresentado por essas mulheres, reiterando o resultado da entrevista.

Podemos observar, por meio da avaliação do estado depressivo, no presente estudo que além destas mulheres, as voluntárias do GC também apresentaram estados depressivos em escala semelhante e em alguns casos maior que no GM. Isto pode ter ocorrido porque, embora as voluntárias do GC não tenham passado pela experiência emocional dolorosa da retirada da mama ou de parte dela, elas possuem seus próprios problemas sociais.

\section{Segundo Kaplan e Sadock $^{15}$, a} depressão está entre os transtornos psiquiátricos mais comuns dos adultos, e tem como característica o humor deprimido, alteração no apetite, perturbação do sono, energia reduzida, cansaço, fadiga, motivação diminuída e ansiedade. Pode ainda haver pensamentos sobre morte, ideação suicida ou tentativa de suicídio. Existem relatos de que aproximadamente dois terços dos pacientes depressivos pensam em suicídio, e que 10 a $15 \%$ o cometem. Alguns indivíduos relatam queixas somáticas e irritabilidade aumentada (por exemplo, raiva persistente), que podem ser percebidas a partir da expressão facial. Dentre as alterações psicomotoras, estão incluídas agitação ou retardo psicomotor, que é mais comum como, por exemplo, pensamento ou movimentos corporais mais lentos. A apresentação de um paciente depressivo é a de alguém com postura curvada sem movimentos espontâneos e com um olhar abatido, desviado ${ }^{5}$.

Diante do exposto, em um terceiro momento o presente estudo associou o estado depressivo de todas as voluntárias à postura corporal por elas adquirida. Os dados evidenciam que as voluntárias classificadas como depressivas de grau moderado à grave, apresentam principalmente uma postura corporal com inclinação de cabeça para esquerda, elevação de ombro para direita ou para esquerda (mesma incidência), flexão e anteriorização de cabeça, assimetria de ambas as escápulas, inclinação posterior de tronco e retroversão pélvica. Algumas dessas alterações também são apresentadas por Canales $^{5}$ como uma postura corporal resultante dos sinais e sintomas da depressão em grau severo. 
Como método de avaliação postural utilizado neste estudo, a fotogrametria computadorizada mostrou ser uma ferramenta eficaz em destacar resultados abrangentes nos dois grupos participantes, tanto isoladamente quanto de forma global. Lunes et. al. ${ }^{16}$ confirmaram a eficácia deste método ao realizar um estudo comparando o método de avaliação postural visual com a fotogrametria computadorizada e, segundo os autores, a fotogrametria foi considerada capaz de detectar mais precisamente as assimetrias, sendo 0 método mais concordante entre examinadores diferentes, quando se compara com a avaliação postural visual.

\section{CONCLUSÃO}

Pode-se concluir que as alterações posturais estiveram presentes tanto nas voluntárias do GM quanto nas do GC, porém ao comparar a quantidade de alterações presentes em ambos os grupos concluiu-se que não houve diferença significativa entre os mesmos.

Foi observado que os indivíduos que se enquadraram como depressivos em grau moderado ou grave apresentaram mais alterações posturais. Desta forma, esta pesquisa reitera a importância da realização periódica de análise postural de forma eficaz pode ser realizada por meio do Software para $\begin{array}{llll}\text { Avaliação Postural (SAPO) e o } & \end{array}$ acompanhamento do nível de depressão, pois ambos podem ajudar a direcionar a forma de tratamento .

Ficou evidenciado que, tanto mulheres mastectomizadas, quanto as não mastectomizadas apresentam alterações posturais e possuem quadros depressivos semelhantes. Portanto, não há uma relação direta entre alteração postural e depressão em mulheres mastectomizadas.

\section{DECLARAÇÃO DE CONFLITO DE INTERESSE}

Os autores declaram não haver qualquer potencial conflito de interesse que possa interferir na imparcialidade deste trabalho científico.

\section{REFERÊNCIAS}

1. Batiston AP, Santiago SM. Fisioterapia e complicações físico-funcionais após tratamento cirúrgico do câncer de mama. Fisioter Pesq. 2005;12(3):30-5.

2. Rostkowska E, Bak M, Samborski W. Body posture in women after mastectomy and its changes as a result of rehabilitation. Adv Med Sciences (Poland). 2006;51:287-97.

3. Lima IA, Vendramini T. Reeducação postural global em pacientes mastectomizadas. Rev Fisiobrasil 2006;78(2):7-13.

4. Pereira OS. A utilização da análise computadorizada como método de avaliação das alterações posturais: um estudo preliminar. Fisiot Mov. 2003;16(2):17-25.

5. Braz RG, Goes FPD, Carvalho GA. Confiabilidade e validade de medidas 
angulares por meio do software para avaliação postural. Fisiot Mov. 2008;21(3):117-26.

6. Iunes DH, Castro FA, Salgado HS, Moura IC, Oliveira AS. Bevilaqua-Grossi D. Confiabilidade intra e interexaminadores e repetibilidade da avaliação postural pela fotogrametria. Rev Bras Fisioter. 2005;9(3):327-334.

7. Melo MSI, Maia JN, Silva DAL, Carvalho CC. Avaliação Postural em Pacientes Submetidas à Mastectomia Radical Modificada por meio da Fotogrametria Computadorizada. Rev Bras Cancerol. 2011;57(1):39-48.

8. Gouveia PF, Gonzáles EO, Grer PA, Fernandes CA, Lima MC. Avaliação de amplitude de movimento e força da cintura escapular em pacientes de pós-operatório tardio de mastectomia radical modificada. Fisioter Pesq São Paulo. 2008;15(2):172-6. DOI: $\quad$ http://dx.doi.org/10.1590/S1809$\underline{29502008000200010}$

9. Canales JZ, Cordás TA, Fiquer JT, Cavalcante AF, Moreno RA. Posture and body image in individuals with major depressive disorder: a controlled study. Rev Bras Psiquiat. 2010;32:375-80. DOI: http://dx.doi.org/10.1590/S1516$\underline{44462010000400010}$

10. Beck AT, Ward $\mathrm{CH}$, Mendelson M, Mock $\mathrm{J}$, Erbaugh J. An Inventory for Measuring Depression. Arch Gen Psychiatry. 1961;4:5363. DOI: http://dx.doi.org/10.1001/archpsyc.1961.017 $\underline{10120031004}$

11. Cody RP. Applied Statistics and the SAS programming language. New York, 1987.

12. Cardoso FR, Oliveira F. Avaliação postural em mulheres submetidas à mastectomia radical modificada: estudo de cinco casos. Rev Lat Mastol. 2004;5(1):14-18.
13. Silva JA, Rodrigues RS. Análise do comportamento articular do ombro homolateral em pacientes submetidas a mastectomia radical modificada. Universidade Castelo Branco. 2007;6:1-13.

14. Ferreira NRC, Kappaun MEC. A imagem corporal de mulheres mastectomizadas. HU Rev. 2008;34(4):243-8.

15. Kaplan HI, Sadock BJ. Tratado de psiquiatria. Artes Médicas. Porto Alegre. 1999.

16. Iunes $\mathrm{DH}$, Bevilaqua-Grossi $\mathrm{D}$, Oliveira AS, Castro FA, Salgado HS. Análise comparativa entre avaliação postural visual e por fotogrametria computadorizada. Rev Bras Fisioter. 2009;13(4):308-15. DOI: http://dx.doi.org/10.1590/S1413$\underline{35552009005000039}$

Recebido para publicação em 06/08/2013 Revisado em 12/09/2014

Aceito em 17/10/2014 